\title{
When does atomic resolution plan view imaging of surfaces work?
}

\author{
Pratik Koirala $^{1}$, Yuyuan Lin ${ }^{1}$, Jim Ciston $^{2}$ and Laurence D. Marks ${ }^{1 *}$ \\ ${ }^{1}$ Department of Materials Science and Engineering, Northwestern University, Evanston, IL \\ 60208, USA. \\ ${ }^{2}$ National Center for Electron Microscopy, The Molecular Foundry, Lawrence Berkeley National \\ Laboratory, Berkeley, California 94720, USA.
}

\begin{abstract}
Surface structures that are different from the corresponding bulk, reconstructions, are exceedingly difficult to characterize with most experimental methods. Scanning tunneling microscopy, the workhorse for imaging complex surface structures of metals and semiconductors, is not as effective for oxides and other insulating materials. This paper details the use of transmission electron microscopy plan view imaging in conjunction with image processing for solving complex surface structures. We address the issue of extracting the surface structure from a weak signal with a large bulk contribution. This method requires the sample to be thin enough for kinematical assumptions to be valid. The analysis was performed on two sets of data, $\mathrm{c}(6 \times 2)$ on the $(100)$ surface and $(3 \times 3)$ on the (111) surface of $\mathrm{SrTiO}_{3}$, and was unsuccessful in the latter due to the thickness of the sample and a lack of inversion symmetry. The limits and the functionality of this method are discussed.
\end{abstract}

*Corresponding author 


\section{Introduction}

The challenge of extracting a signal with low intensity from a projection with other strong signals has always been pertinent to the field of signal processing. A similar challenge exists in the surface science community to extract the surface structure from one with a large bulk component. Several techniques have been developed both from the theoretical and experimental fronts to address this issue. For conducting materials and simple unit cells, low-energy electron diffraction (LEED) is a very powerful approach [1-6], particularly when complemented by atomic resolution scanning probe methods [4, 7-23]. Transmission electron diffraction (TED) $[24,25]$ in unison with direct methods [26-29], X-ray scattering studies [29-31], reflection high energy electron diffraction (RHEED) [20, 32, 33] and recently high resolution secondary electron microscopy (HRSEM) [34] have also been effectively used to study surface structures. In many cases, these methods are complementary.

With more complex reconstructions and also insulators, many of these techniques have very severe limitations. This is particularly relevant for oxide materials which have an abundance of surface reconstructions; even the prototypical perovskite material $\mathrm{SrTiO}_{3}$ has highly complex surface structures $[1,2,4,6,9,12-14,16,17,19,21-25,27,29,34-37]$. The surfaces of these oxides are of prime importance as many phenomena occur at the surface. For instance, the 2D electron gas $[38,39]$ at the $\mathrm{LaAlO}_{3} / \mathrm{SrTiO}_{3}$ interface is a direct consequence of the interfacial structures of the two oxides. This paper presents plan view high resolution transmission electron microscopy as a viable approach for imaging complex surface structures and the complexities therein. 
Transmission electron microscopy (TEM) is a powerful technique for studying complex surfaces due to its high signal to noise ratio. TEM is used in two different modes, plan view [40-50] and profile view [48, 49, 51-71], with respect to the orientation of the sample surface. Imaging of nanoparticle surfaces is more suited to profile view imaging as has been demonstrated for gold and silver particles [51-63] and more recently for oxide nanoparticles [72, 73]. It can give out of plane relaxations but includes little to no information along the beam direction. On the other hand, plan view imaging provides two-dimensional information in the plane of the surface, although no information normal to the surface, and has been previously used to solve two highly complex surface reconstruction $[34,41]$.

In plan view one has surface structures on both top and bottom surfaces, and must extract the single surface information to move forward. The approach used to date is to assume a kinematical model and linear imaging theory, and consider the image after bulk removal to be a simple addition of the top and bottom surface [41] as:

$$
\begin{gathered}
\Psi(\mathbf{r})=1+\boldsymbol{\sigma}[\mathrm{V}(\mathbf{r})+\mathrm{V}(\mathbf{d}-\mathbf{r})] \\
\mathrm{I}(\mathbf{r})=1+\int \boldsymbol{\sigma} \mathrm{T}(\mathrm{u})\left\{\mathrm{V}(\mathrm{u})+\mathrm{V}^{*}(\mathbf{u}) \exp (2 \pi \mathrm{id} . \mathbf{u})\right\} \times \exp (-2 \pi \mathrm{iu} . \mathbf{r}) d \mathrm{u}+\eta(\mathbf{r})
\end{gathered}
$$

where, $\mathrm{V}(\mathbf{r})$ and $\mathrm{V}(\mathbf{d}-\mathbf{r})$ are the potentials of the top and bottom surface respective, $d$ is the in plane translation vector between the top and bottom surfaces, $\boldsymbol{\sigma}$ is the relativistic interaction constant, $T(\mathbf{u})$ accounts for the microscope parameters and $\eta(\mathbf{r})$ is the noise in the image. For completeness, we note that without the bulk component this is not a true "image" of the surface, rather a surface sensitive difference closer to a difference map in a conventional crystallographic sense. One deals with the same type of signal when using direct methods for surfaces, and the 
loss of the bulk component in general has not proved to be an issue in interpreting the maps [2629, 74].

While this method can work [34, 41], it ignores dynamical diffraction coupling with the bulk (e.g. $[44,50,75-78]$ ) and the limitations and functionality of this method have not been analyzed in detail to date. In this paper, we discuss this in more detail, showing that the method is only robust when the surface contains inversion symmetry.

\section{Methods}

\subsection{Sample Preparation}

Self-supported single crystal samples were prepared from bulk (100) and (111) $\mathrm{SrTiO}_{3}$ substrates purchased from MTI Corporation (Richmond, CA). They were cut into $3 \mathrm{~mm}$ discs using an ultrasonic cutter, mechanically thinned to a thickness of $\sim 100 \mu \mathrm{m}$ using silicon carbide sandpaper, then dimpled with a Gatan 656 Dimple Grinder and $0.5 \mu \mathrm{m}$ diamond slurry until the thickness at the center was $\sim 30 \mu \mathrm{m}$. The samples were then washed with de-ionized water, soaked in acetone overnight and finally cleaned with methanol. The samples were then $\mathrm{Ar}^{+}$ion milled to electron transparency using a Gatan Precision Ion Polishing System (PIPS) starting at an energy of $5 \mathrm{keV}$ and milling angle of $10^{\circ}$. The ion energy and milling angle were gradually brought down to $3 \mathrm{keV}$ and $6^{\circ}$ respectively for final polishing and surface cleaning.

The samples were then annealed in flowing dry oxygen at $1050-1200^{\circ} \mathrm{C}$ for $10 \mathrm{~h}$ in a quartz tube furnace. Both samples were baked in air at $300-500{ }^{\circ} \mathrm{C}$ for $1-4 \mathrm{~h}$ directly before the imaging experiments.

\subsection{Imaging Experiments}


High Resolution TEM (HRTEM) experiments were performed on the TEAM 0.5 instrument (FEI Titan-class) at the National Center for Electron Microscopy (NCEM). The (100) sample with $\mathrm{c}(6 \times 2)$ surface reconstruction was imaged at an accelerating voltage of $80 \mathrm{kV}$ with an energy spread of $0.1 \mathrm{eV}, 0.2 \mathrm{mrad}$ convergence angle, $1.4 \mathrm{~nm}$ defocus spread and the aberration corrector tuned to balance $C_{3}$ against the uncorrected residual $C_{5}\left(C_{3}=-16 \mu \mathrm{m}, C_{5}=6 \mathrm{~mm}\right)$. A focal series of 41 images was acquired at defocus steps of $-1.05 \mathrm{~nm}$. The focal series was used for determining the appropriate defocus to maximally enhance bulk subtraction.

The (111) sample with the $(3 \times 3)$ surface reconstruction was imaged at an accelerating voltage of $300 \mathrm{kV}$ with an energy spread of $0.1 \mathrm{eV}, 0.15 \mathrm{mrad}$ convergence angle, $0.7 \mathrm{~nm}$ defocus spread and the aberration corrector tuned to balance $C_{3}$ against the uncorrected residual $C_{5}\left(C_{3}=-15 \mu \mathrm{m}\right.$, $\mathrm{C}_{5}=6 \mathrm{~mm}$ ). A focal series of 41 images was acquired at defocus steps of $-1.72 \mathrm{~nm}$. No significant beam damage was observed in either of the two samples.

\subsection{Simulations and Post Processing}

High resolution TEM simulations were performed using MacTempasX Code [79] with experimental parameters and the post processing of both experimental and simulated images (see Supplemental information for the Crystallographic Information File c(6x2).cif), bulk removal and correction for top and bottom surface translation, was done using the in house open source code Electron Direct Methods (EDM) [80].

\section{Results}

High resolution plan view images acquired on two sets of reconstructions on the (001) and (111) surfaces from the same material, $\mathrm{SrTiO}_{3}$, were subject to image analysis outlined in the methods section. Two model cases, one demonstrating a successful use of the aforementioned method and 
one a failure, are discussed to better present the functionality of the analysis pertaining to two important parameters:

1. Sample thickness, discussed with $\mathrm{c}(6 \times 2)$ on $\mathrm{SrTiO}_{3}(001)$ and the $(3 \times 3)$ on $\mathrm{SrTiO} 3$ (111) as a model cases

2. Symmetry, discussed with $(3 \times 3)$ on $\mathrm{SrTiO}_{3}(111)$ as a model case

\section{$3.1 \mathrm{c}(6 \times 2)$ surface reconstruction on $\mathrm{SrTiO}_{3}(001)$ surface}

High resolution plan view images in a focal series of 41 images were processed using the method outlined in the previous section. The consistency of experimental parameters and defocus were cross checked with simulations from MacTempas. Removal of bulk was done by taking a fast Fourier transform of an image and removing all linear combination of the bulk reciprocal lattice vectors. An unavoidable consequence of this is that the overlapping surface and bulk spots are removed so this is strictly a difference map as mentioned earlier. This was followed by the separation of the top and bottom surface. The resulting image for the surface structure acquired from one of the experimental image of defocus $6 \AA$ is given in Figure 1 along with the corresponding DFT relaxed structure.

In the limits of linear imaging theory, the resulting image can be directly correlated to the intensity of different atomic sites at the surface. The corresponding structure of the $c(6 \times 2)$ surface reconstruction (see Figure 1) is consistent with atomic resolution secondary electron images [34] as well as x-ray and scanning tunneling microscopy[4] data. The details of the actual structure and surface chemistry are discussed elsewhere[34]. 


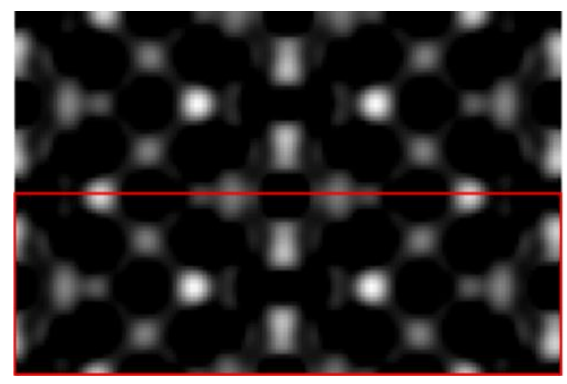

(a)

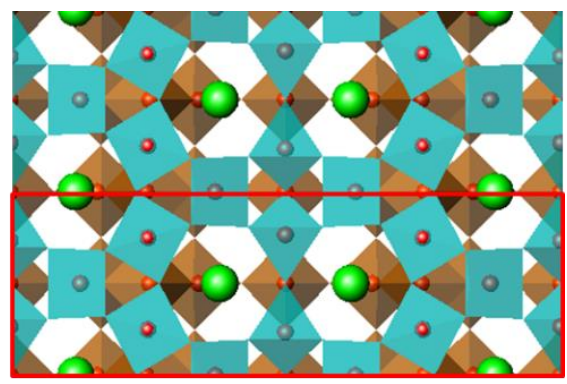

(b)

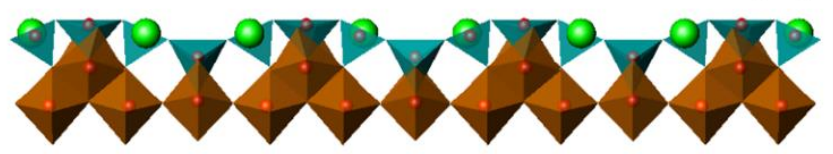

(c)

Figure 1. In (a) experimental image of the $c(6 \times 2)$ surface reconstruction after the removal of bulk and separation of top and bottom surface from high resolution plan view transmission electron microscopy image and the corresponding structure in (b) plan view and (c) profile view. (The unit cell is outlined in red.)

It is evident from Figure 1 that the method used for the extraction of surface structure is effective. The correlation between the actual structure in Figure $1 \mathrm{~b}$ and the relative intensities in the experimental image is strong. The position of $\mathrm{Sr}$ atoms appear relatively brighter on the experimental image, consistent with Sr being heavier than Ti and O. Separation performed on all 41 images in the focal series show strong intensity at the Sr position with small modulations in the rest of the image.

Simulations were performed using MacTempas with the structure in Figure 1(b) for varying bulk thicknesses. The results of the analysis performed at four different thicknesses and hence different bulk contributions and dynamical scattering are given in Figure 2. Since this method relies on linear imaging theory, there is a critical thickness beyond which the approximations are no longer valid. This critical thickness is dependent on the materials property for instance this 
method would work for relatively thicker samples of silicon in comparison to strontium titanate $\left(\mathrm{SrTiO}_{3}\right)$.

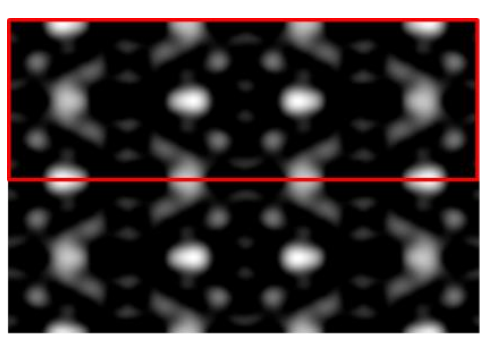

(a)

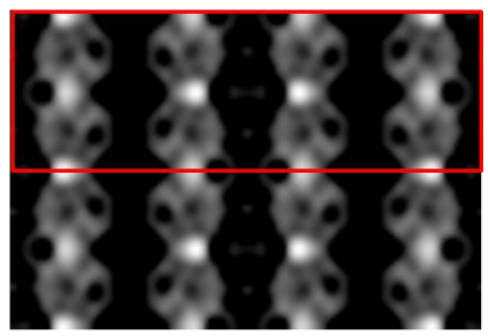

(c)

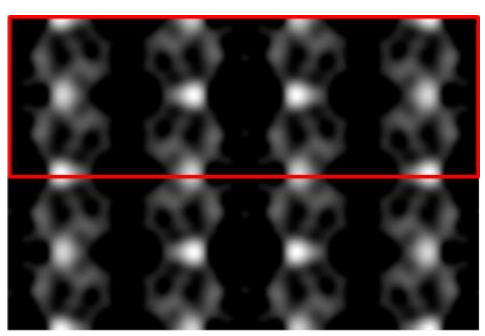

(b)

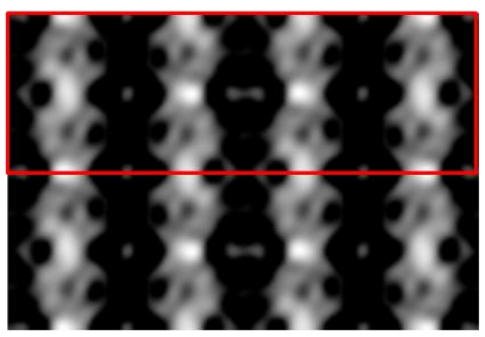

(d)

Figure 2. Simulated plan view images after bulk removal (defocus=6 $\AA$ ) at thicknesses of (a) $4.15 \mathrm{~nm}$, (b) $5.32 \mathrm{~nm}$, (c) $6.49 \mathrm{~nm}$ and (d) $7.66 \mathrm{~nm}$. Vibration of $0.4 \AA$ (root mean squared) in the $\mathrm{x}$ - and $\mathrm{y}$-directions has been added to all images to mimic experimental conditions. (The $\mathrm{c}(6 \times 2)$ unit cell is outlined.)

Surface signal is highly sensitive to the thickness of the sample. Images simulated at $4.15 \mathrm{~nm}$ and $5.32 \mathrm{~nm}$ thickness show a strong surface signal evident after bulk subtraction. However, the images simulated at $6.49 \mathrm{~nm}$ and $7.66 \mathrm{~nm}$ thickness show weak surface signal. This is a clear demonstration that the thickness of the sample has to be in the kinematical regime, which for the case of $\mathrm{SrTiO}_{3}$ is approximately $5 \mathrm{~nm}$. The useable, kinematical thickness will vary with respect to the orientation, accelerating voltage and aberrations. For instance, the (111) orientation of 
$\mathrm{SrTiO}_{3}$ has a higher in plane atomic density than the (100), thus resulting in an even smaller thickness for the kinematical approximation to be valid.

In the case of $c(6 \times 2)$, the presence of an inversion center allows for a more robust separation of the top and bottom surface unlike in the case of the $(3 \times 3)$ surface reconstruction on $\mathrm{SrTiO}_{3}(111)$ surface. This in large part because the phase can only be 0 or 180 degrees with inversion symmetry. It is worth remembering that the phase is generally more important than the amplitude in obtaining representative images.

\section{2 (3×3) surface reconstruction on $\mathrm{SrTiO}_{3}(111)$}

The separation of the top and bottom surface was done in two different plane group symmetries: $\mathrm{p} 6 \mathrm{~mm}$ and $\mathrm{p} 3 \mathrm{~m} 1$. While the actual structure is $\mathrm{p} 3 \mathrm{~m} 1$, the lack of inversion symmetry in $\mathrm{p} 3 \mathrm{~m} 1$ makes it difficult to interpret the resulting image. We note that it is well established with direct methods of inverting diffraction data that sometimes higher symmetry space groups with inversion symmetry solve better, particularly if the symmetry reduction of the true structure is small; this was also found for surfaces [74].

Separation of the top and bottom surface was performed on the same experimental image taken at a defocus of $5.4 \mathrm{~nm}$. The resulting images in $\mathrm{p} 6 \mathrm{~mm}$ and $\mathrm{p} 3 \mathrm{~m} 1$ after the separation are given in Figures $3 \mathrm{a}$ and $3 \mathrm{~b}$, respectively. One common feature that is consistent across both the images is the high signal at the origin. In contrast, other experimental data including STM and density functional theory based calculations [81] (see Figures 3c and 3d) show the opposite. This can be attributed to relatively thick samples, thicker than the sample on which the $c(6 \times 2)$ was observed. As seen in Figure 2, the restrictions on the thickness of the sample is strict and the higher in 
plane atomic density along (111) in comparison to the (001) direction is detrimental to the validity of kinematical assumptions.

In addition to the inconsistency at the origin, the $\mathrm{p} 3 \mathrm{~m} 1$ image in general does not match with the experimental STM image making it almost impossible to interpret the contrast or the atomic positions. On the contrary, the image in $\mathrm{p} 6 \mathrm{~mm}$ provides significant details that are consistent with the STM image and the structure. However, since the actual structure is not p6mm the intensities are deviant. The six membered ring at the origin is rotated by $30^{\circ}$. However, besides the rotation of the six membered ring and strong intensity at the origin, the p6mm image provides a better match with the experimental image, thus demonstrating the importance of an inversion center.

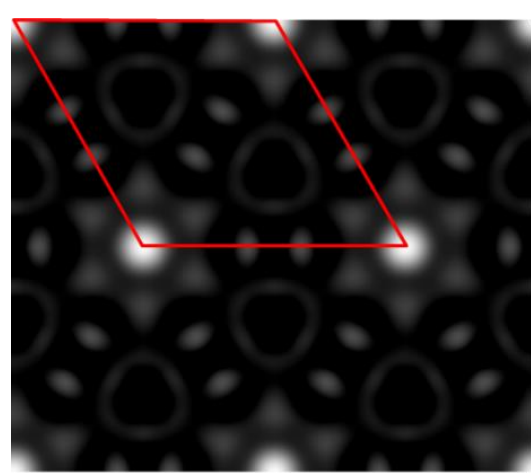

(a)

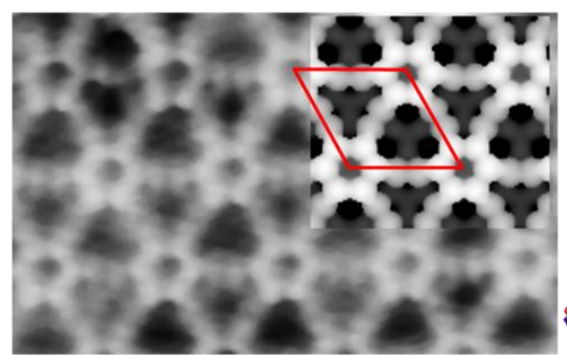

(c)

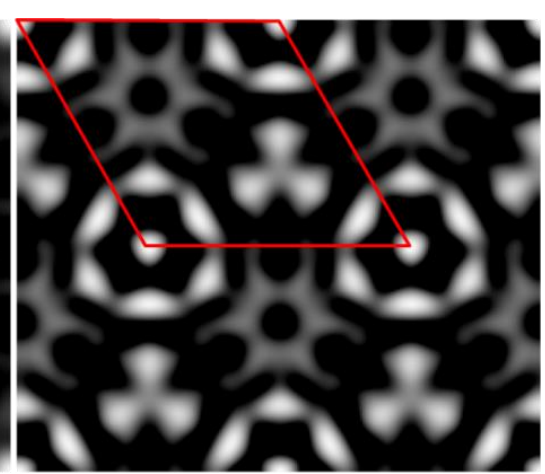

(b)

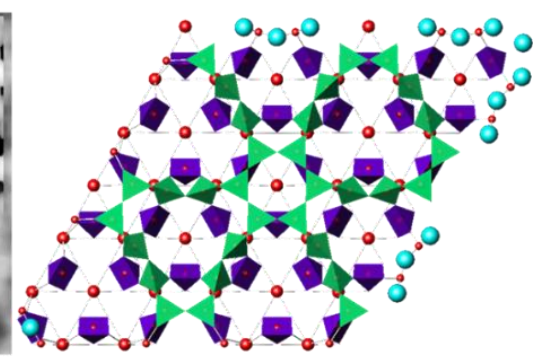

(d)

Figure 3. Experimental HREM image in (a) with p6mm symmetry and in (b) with p3m1 symmetry used for the separation of top and bottom surface after bulk removal. In (c) STM 
image with the simulation overlaid and (d) the corresponding structure of the $(3 \times 3)$ surface reconstruction on $\mathrm{SrTiO}_{3}$ on (111) [Subfigures (c) and (d) adapted from Ref. [81].] (The (3×3) unit cell is outlined in red.)

This was validated with HREM simulations performed on the $(3 \times 3)$. The simulations were performed with $\mathrm{S} 3 \times 3$.cif (see supplemental materials) using the experimental parameters given in the methods section.

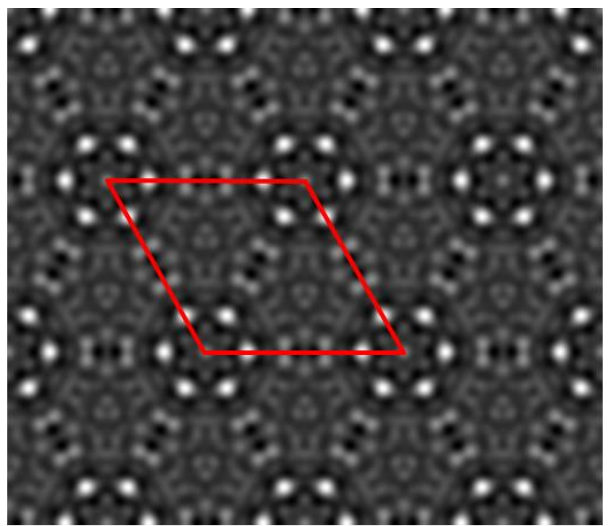

(a)

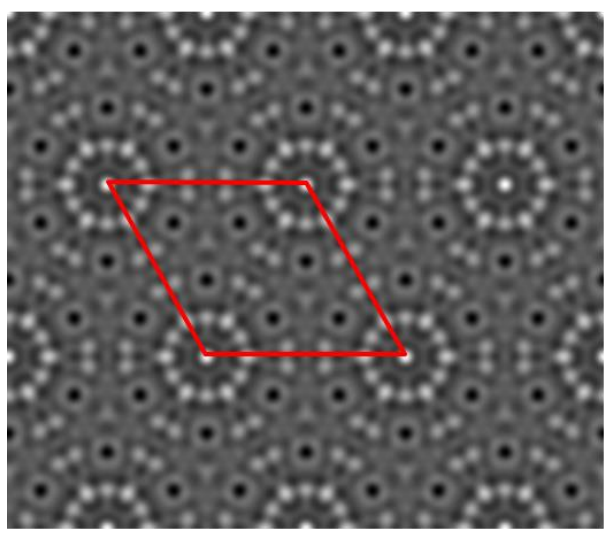

(c)

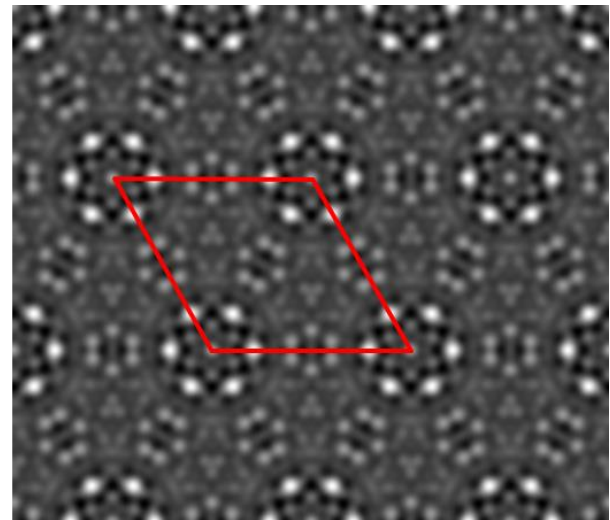

(b)

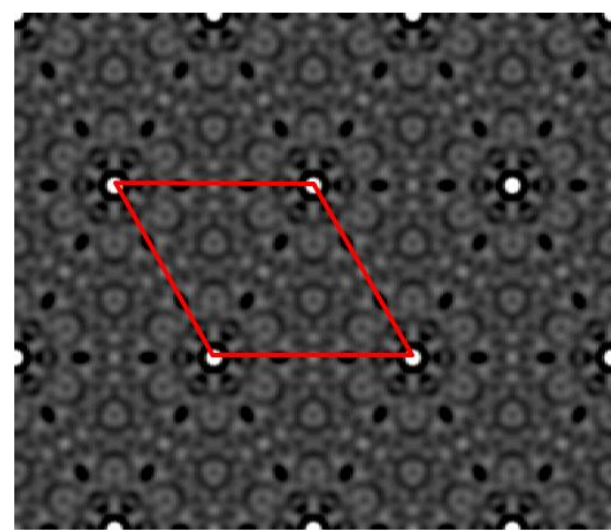

(d)

Figure 4. Simulated HREM images of the $(3 \times 3)$ surface reconstruction on $\mathrm{SrTiO}_{3}(111)$ after bulk removal at varying thicknesses in (a) $5 \mathrm{~nm}$, (b) $6.33 \mathrm{~nm}$, (c) $7.7 \mathrm{~nm}$ and (d) $9.07 \mathrm{~nm}$. (The $(3 \times 3)$ surface unit cell is outlined in red.) 
The high intensity observed at the origin in the experimental plan view images is only seen in images of at least $6 \mathrm{~nm}$ thickness; the intensity at the origin is stronger in images simulated at increasing thickness. Hence, the high intensity at the origin observed in the experimental image is the result of dynamical effects in thicker sample. In addition, the rotation of the six membered ring is not present in the simulated image below a thickness of $6 \mathrm{~nm}$. However, the image simulated at $7.7 \mathrm{~nm}$ thickness starts to show intensity that is consistent with the $30^{\circ}$ rotation of the hexagonal ring. Finally, the image simulated at a thickness of $9.07 \mathrm{~nm}$ shows the rotation of the six membered ring consistent with the inversion performed on experimental images. This further validates that the sample in the case of $(3 \times 3)$ was in the tens of $\mathrm{nm}$.

For completeness, experimental images on the two extremes of the focal series were also analyzed to compare the separation under $\mathrm{p} 6 \mathrm{~mm}$ and $\mathrm{p} 3 \mathrm{~m} 1$. This is particularly important if there is inversion of phases during the separation and in some cases the resulting solution could be an example of Babinet's principle, i.e. the inverse solution. The contrast on the two ends of the focal spectrum clearly demonstrate that the intensities are still consistent in p6mm. However, in the case of $\mathrm{p} 3 \mathrm{~m} 1$ there is a dramatic change in contrast. This can also be attributed to the presence of inversion center in p6mm thus making the intensities symmetric on both sides of the focal series. 


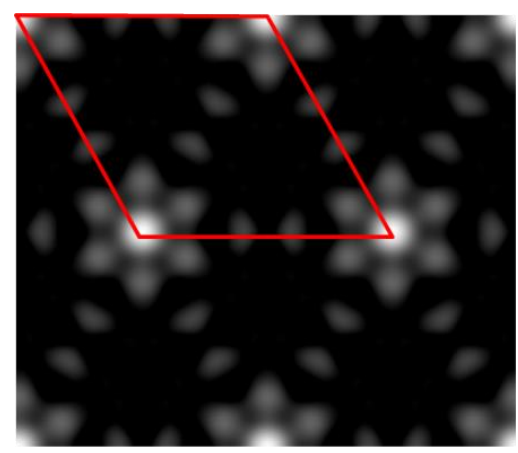

(a)

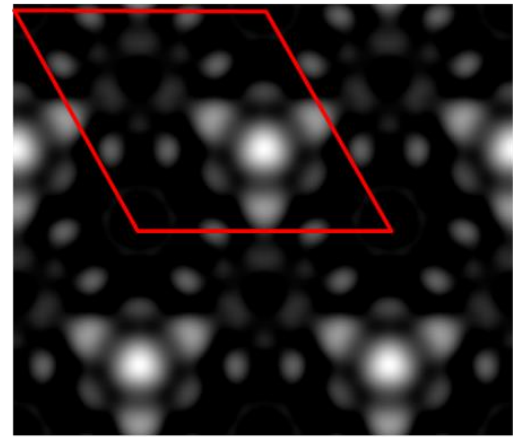

(c)

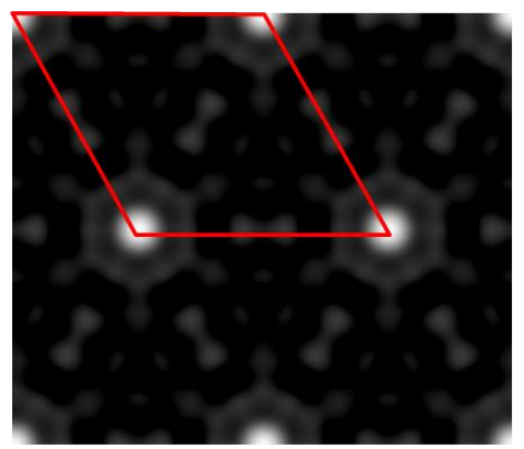

(b)

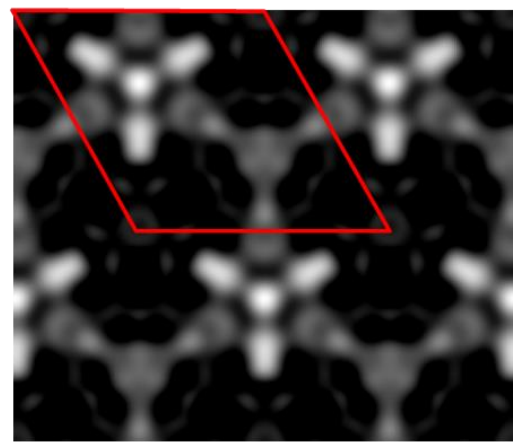

(d)

Figure 5. Experimental images after bulk removal and separation of top and bottom surface: with p6mm symmetry in (a) and (b) with defocus of experimental image at $41.3 \mathrm{~nm}$ and -27.5 $\mathrm{nm}$ respectively and with $\mathrm{p} 3 \mathrm{~m} 1$ symmetry in (c) and (d) with defocus of experimental image at $41.3 \mathrm{~nm}$ and $-27.5 \mathrm{~nm}$ respectively.

\section{Discussion}

The primary limitation of the inversion method lies in the assumptions that the scattering is in the kinematical regime which as we have seen in the case of the $(3 \times 3)$ is not always valid. Implementation of dynamical scattering theory would be essential going beyond surface structures with inversion symmetry or a thin sample.; while this has been done to refine surface diffraction data (e.g. [44, 82-84]), it is rather tedious and it is unclear how to do this simply. Another challenge would be to account for differences in the top and bottom surface. There can 
be coexisting domains as well as different overlapping periodicities. While the inversion may still work if the surface structure of concern occupies a majority of the surface area as the plan view method works with the average of domains, this is not always the case. This gives rise to inconsistency in the results even for images acquired from the same sample but from different areas. For instance, different $(\mathrm{n} \times \mathrm{n})$ reconstructions have been found to coexist on the $\mathrm{SrTiO}_{3}$ (111) surface along with other difficulties due to long range disorder in the sample and glass like behavior of some of the surfaces [81]. Similarly, issues with the signal to noise $(\mathrm{S} / \mathrm{N})$ ratio from the surface is critical. It could well be that beyond a certain thickness, the $\mathrm{S} / \mathrm{N}$ from the surface is insufficient for extracting any useful information.

Plan view imaging possesses good surface signal, $1-3 \%$ of the total signal, if the sample is thin. This method has been used for four systems, $(7 \times 7)$ on silicon (111) [41], 5x2-Au on silicon (111)

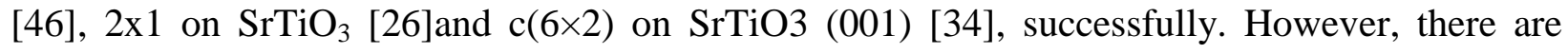
restrictions, the first being on the thickness of the sample. The second requirement which is subtler pertains to having inversion symmetry. The requirements for inversion symmetry can be thought of as imposing restriction on the sets of phases for different reflections thus making the method more robust and the resulting intensities more reliable. Thus, plan view imaging on the zone axis is a powerful tool when applied to imaging surface structures with proper address to the limitations and functionality of the method. An alternative is to tilt off the zone axis which will reduce dynamical effects, but introduces additional issues as the signal is weaker [50].

Acknowledgement PK acknowledges funding by the Department of Energy on Grant Number DE-FG02-01ER45945. Electron microscopy was performed at the Molecular Foundry at Lawrence Berkeley National Lab, which is supported by the Office of Basic Energy Sciences of the US Department of Energy under Contract No. DE-AC02-05CH11231. 


\section{References:}

[1] J.E.T. Andersen, P.J. Mo $\square$ ller, Impurity-induced $900{ }^{\circ} \mathrm{C}(2 \times 2)$ surface reconstruction of $\mathrm{SrTiO}_{3}(100)$, Applied Physics Letters, 56 (1990) 1847.

[2] Y. Cao, S. Wang, S. Liu, Q. Guo, J. Guo, Electronic structures of the $\mathrm{SrTiO}_{3}(110)$ surface in different reconstructions., The Journal of chemical physics, 137 (2012) 044701.

[3] M.S. Gonzalez, M.H. Aguirre, E. Mora, M.C. Asensio, In situ reduction of (100) $\mathrm{SrTiO}_{3}$, Solid State Sciences, 2 (2000) 519-524.

[4] D. Jiang, J. Zegenhagen, c(6x2) and c(4x2) reconstructions of the $\mathrm{SrTiO}_{3}$ (001), Surface Science, 425 (1999) 343-354.

[5] P.J. Moller, A. Komolov, F. Lazneva, Selective growth of a MgO (100) -c (2x2) superstructure on a $\mathrm{SrTiO}_{3}(100)-(2 \times 2)$ substrate, Surface Science, 425 (1999) 15-21.

[6] R. Shimizu, K. Iwaya, T. Ohsawa, S. Shiraki, T. Hasegawa, T. Hashizume, T. Hitosugi, Effect of oxygen deficiency on $\mathrm{SrTiO}_{3}$ (001) surface reconstructions, Applied Physics Letters, 100 (2012) 263106.

[7] T. Beck, A. Klust, M. Batzill, U. Diebold, C. Di Valentin, A. Selloni, Surface Structure of $\mathrm{TiO}_{2}(011)-(2 \times 1)$, Physical Review Letters, 93 (2004) 036104.

[8] M.R. Castell, Nanostructures on the $\mathrm{SrTiO}_{3}$ (001) surface studied by STM, Surface Science, 516 (2002) 33-42.

[9] M.R. Castell, Scanning tunneling microscopy of reconstructions on the $\mathrm{SrTiO}_{3}(001)$ Surface, Surface Science, 505 (2002) 1-13.

[10] D.S. Deak, F. Silly, D.T. Newell, M.R. Castell, Ordering of $\mathrm{TiO}_{2}$-Based Nanostructures on $\mathrm{SrTiO}_{3}(001)$ Surfaces., The Journal of Physical Chemistry B, 110 (2006) 9246-9251.

[11] D.S. Deak, F. Silly, K. Porfyrakis, M.R. Castell, Controlled surface ordering of endohedral fullerenes with a $\mathrm{SrTiO}_{3}$ template., Nanotechnology, 18 (2007) 075301.

[12] J. Feng, X. Zhu, J. Guo, Reconstructions on $\mathrm{SrTiO}_{3}(111)$ surface tuned by Ti/Sr deposition, Surface Science, 614 (2013) 38-45.

[13] K. Johnston, M. Castell, A. Paxton, M. Finnis, $\mathrm{SrTiO}_{3}(001)(2 \times 1)$ reconstructions: First-principles calculations of surface energy and atomic structure compared with scanning tunneling microscopy images, Physical Review B, 70 (2004) 085415.

[14] T. Kubo, H. Nozoye, Surface structure of $\mathrm{SrTiO}_{3}(100)$, Surface Science, 542 (2003) 177-191.

[15] T. Kubo, H. Nozoye, Surface Structure of $\mathrm{SrTiO}_{3}(100)-(\sqrt{ } 5 \times \sqrt{ } 5)-R 26.6^{\circ}$, Physical Review Letters, 86 (2001) 1801-1804.

[16] Y. Lin, A.E. Becerra-Toledo, F. Silly, K.R. Poeppelmeier, M.R. Castell, L.D. Marks, The (2×2) reconstructions on the $\mathrm{SrTiO}_{3}(001)$ surface: A combined scanning tunneling microscopy and density functional theory study, Surface Science, 605 (2011) L51-L55.

[17] L.D. Marks, a.N. Chiaramonti, S.U. Rahman, M.R. Castell, Transition from Order to Configurational Disorder for Surface Reconstructions on $\mathrm{SrTiO}_{3}$ (111), Physical Review Letters, 114 (2015) 1-6.

[18] H.L. Marsh, D.S. Deak, F. Silly, a.I. Kirkland, M.R. Castell, Hot STM of nanostructure dynamics on $\mathrm{SrTiO}_{3}$ (001). Nanotechnology, 17 (2006) 3543-3548.

[19] D. Newell, A. Harrison, F. Silly, M. Castell, $\mathrm{SrTiO}_{3}(001)-(5 \times 5)-\mathrm{R} 26.6^{\circ}$ reconstruction: A surface resulting from phase separation in a reducing environment, Physical Review B, 75 (2007) 205429.

[20] T. Ohsawa, K. Iwaya, R. Shimizu, T. Hashizume, T. Hitosugi, Thickness-dependent local surface electronic structures of homoepitaxial $\mathrm{SrTiO}_{3}$ thin films, Journal of Applied Physics, 108 (2010) 073710.

[21] B.C. Russell, M.R. Castell, Surface of Sputtered and Annealed Polar $\mathrm{SrTiO}_{3}(111)$ : $\mathrm{TiO}_{x}-\mathrm{Rich}(\mathrm{n} \times \mathrm{n})$ Reconstructions, Journal of Physical Chemistry C, 3 (2008) 6538-6545.

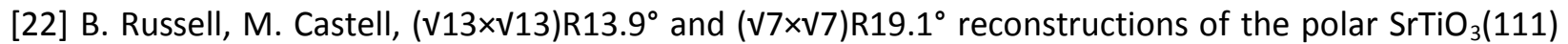
surface, Physical Review B, 75 (2007) 155433. 
[23] F. Silly, D.T. Newell, M.R. Castell, $\mathrm{SrTiO}_{3}(001)$ reconstructions: the $(2 \times 2)$ to $\mathrm{c}(4 \times 4)$ transition, Surface Science, 600 (2006) L219-L223.

[24] A.N. Chiaramonti, C.H. Lanier, L.D. Marks, P.C. Stair, Time, temperature, and oxygen partial pressure-dependent surface reconstructions on $\mathrm{SrTiO}_{3}(111)$ : A systematic study of oxygen-rich conditions, Surface Science, 602 (2008) 3018-3025.

[25] S. Gerhold, Z. Wang, M. Schmid, U. Diebold, Stoichiometry-driven switching between surface reconstructions on $\mathrm{SrTiO}_{3}(001)$. Surface science, 621 (2014) L1-L4.

[26] N. Erdman, K.R. Poeppelmeier, M. Asta, O. Warschkow, D.E. Ellis, L.D. Marks, The structure and chemistry of the $\mathrm{TiO}_{2}$-rich surface of $\mathrm{SrTiO}_{3}$ (001). Nature, 419 (2002) 55-58.

[27] N. Erdman, O. Warschkow, M. Asta, K.R. Poeppelmeier, D.E. Ellis, L.D. Marks, Surface structures of $\mathrm{SrTiO}_{3}$ (001): a $\mathrm{TiO}_{2}$-rich reconstruction with a $\mathrm{c}(4 \times 2)$ unit cell., Journal of the American Chemical Society, 125 (2003) 10050-10056.

[28] D.M. Kienzle, a.E. Becerra-Toledo, L.D. Marks, Vacant-Site Octahedral Tilings on $\mathrm{SrTiO}_{3}(001)$, the (V13xV13)R33.7 ${ }^{\circ}$ Surface, and Related Structures, Physical Review Letters, 106 (2011) 176102.

[29] C. Lanier, A. van de Walle, N. Erdman, E. Landree, O. Warschkow, A. Kazimirov, K. Poeppelmeier, J. Zegenhagen, M. Asta, L. Marks, Atomic-scale structure of the $\mathrm{SrTiO}_{3}(001)-\mathrm{c}(6 \times 2)$ reconstruction: Experiments and first-principles calculations, Physical Review B, 76 (2007) 045421.

[30] a. Pancotti, N. Barrett, L.F. Zagonel, G.M. Vanacore, Multiple scattering x-ray photoelectron diffraction study of the SrTiO3(100) surface, Journal of Applied Physics, 106 (2009) 034104.

[31] O. Bunk, G. Falkenberg, J.H. Zeysing, L. Lottermoser, R.L. Johnson, Structure determination of the indium-induced Si(111)-(4x1) reconstruction by surface x-ray diffraction, Phys. Rev. B, 59 (1999) 1222812231.

[32] J. Cheng, P. Regreny, L. Largeau, G. Patriarche, O. Mauguin, K. Naji, G. Hollinger, G. Saint-Girons, Influence of the surface reconstruction on the growth of InP on $\mathrm{SrTiO}_{3}(001)$, Journal of Crystal Growth, 311 (2009) 1042-1045.

[33] M. Naito, H. Sato, Reflection High-Energy Electron Diffraction Study on the $\mathrm{SrTiO}_{3}$ Surface Structure, Physica C, 229 (1994) 1-11.

[34] J. Ciston, H.G. Brown, a.J. D’Alfonso, P. Koirala, C. Ophus, Y. Lin, Y. Suzuki, H. Inada, Y. Zhu, L.J. Allen, L.D. Marks, Surface determination through atomically resolved secondary-electron imaging, Nature Communications, 6 (2015) 7358.

[35] F. Bottin, F. Finocchi, C. Noguera, Facetting and $(n \times 1)$ reconstructions of $\operatorname{SrTiO}_{3}(110)$ surfaces, Surface Science, 574 (2005) 65-76.

[36] S.-h. Phark, Y.J. Chang, T. Won Noh, Selective growth of perovskite oxides on $\mathrm{SrTiO}_{3}(001)$ by control of surface reconstructions, Applied Physics Letters, 98 (2011) 161908.

[37] O. Warschkow, M. Asta, N. Erdman, K.R. Poeppelmeier, D.E. Ellis, L.D. Marks, $\mathrm{TiO}_{2}$-rich reconstructions of $\mathrm{SrTiO}_{3}(001)$ : a theoretical study of structural patterns, Surface Science, 573 (2004) 446-456.

[38] S.N. Klimin, J. Tempere, J.T. Devreese, D. van der Marel, Interface Superconductivity in $\mathrm{LaAlO}_{3}-\mathrm{SrTiO}_{3}$ Heterostructures, Physical Review B, 89 (2014) 184514.

[39] A. Ohtomo, H.Y. Hwang, A high-mobility electron gas at the $\mathrm{LaAlO}_{3} / \mathrm{SrTiO}_{3}$ heterointerface., Nature, 427 (2004) 423-426.

[40] D.N. Dunn, P. Xu, L.D. Marks, UHV transmission electron microscopy of $\operatorname{Ir}(001$ ) I. Microstructure of the (1x1) and the reconstructed (5x1) surfaces, Surface Science, 294 (1993) 308-321.

[41] E. Bengu, R. Plass, L. Marks, T. Ichihashi, P. Ajayan, S. lijima, Imaging the Dimers in Si(111)-(7x7). Physical review letters, 77 (1996) 4226-4228.

[42] S. Li, R. Li, R. Guan, H. Ye, J. Zhu, Plan-view imaging of oxygen-induced reconstruction on $\mathrm{Ag}(110)$ surface. I. The possibility of imaging surface oxygen, Journal of Electron Microscopy, 49 (2000) 163-172. 
[43] L.D. Marks, Registry and UHV Transmission Electron Diffraction of Surfaces, Ultramicroscopy, 45 (1992) 145-154.

[44] L.D. Marks, P. Xu, D.N. Dunn, UHV Transmission Electron-Microscopy of Ir(001) .II. Atomic Positions of the (5x1) Reconstructed Surface from HREM and R- Factor Refinements, Surface Science, 294 (1993) 322-332.

[45] L.D. Marks, Rigor, and plan-view simulation of surfaces, Ultramicroscopy, 38 (1991) 325-332.

[46] L.D. Marks, R. Plass, Atomic Structure of Si(111)-(5x2)-Au from High Resolution Electron Microscopy and Heavy-Atom Holography, Phys. Rev. Lett., 75 (1995) 2172-2175.

[47] P. Xu, L.D. Marks, Intensities of Surface Diffraction Spots in Plan View, Ultramicroscopy, 45 (1992) 155-157.

[48] N. Ikarashi, K. Kobayashi, H. Koike, H. Hasegawa, K. Yagi, Profile and plan-view imaging of reconstructed surface structures of gold, Ultramicroscopy, 26 (1988) 195-203.

[49] M.A. Gribelyuk, P.J.F. Harris, J.L. Hutchison, Plan-view and profile imaging of sulphided platinum particles, Philosophical Magazine Part B, 69 (1994) 655-669.

[50] P. Xu, D. Dunn, J.P. Zhang, L.D. Marks, Atomic Imaging of Surfaces in Plan View, Surface Science, 285 (1993) L479-L485.

[51] L.D. Marks, Direct atomic imaging of solid surfaces I. Image Simulation and Interpretation, Surface Science, 139 (1984) 281-298.

[52] L.D. Marks, D.J. Smith, Direct atomic imaging of solid surfaces II. Gold (111) surfaces during and after in situ carbon etching, Surface Science, 143 (1984) 495-508.

[53] D.J. Smith, L.D. Marks, Direct Atomic Imaging of Solid Surfaces III. Small particles and extended Au surfaces, Ultramicroscopy, 16 (1985) 101-114.

[54] L.D. Marks, D.J. Smith, Direct Atomic Imaging of Solid Surfaces IV. Dislocations on Au(100), Surface Science Letters, 157 (1985) 367-372.

[55] L.D. Marks, Direct Imaging of Carbon-Covered and Clean Gold (110) Surfaces, Phys. Rev. Lett., 51 (1983) 1000-1002.

[56] D.J. Smith, L.D. Marks, Direct lattice imaging of small metal particles, Philosophical Magazine A, 44 (1981) 735-740.

[57] L.D. Marks, V. Heine, D.J. Smith, Direct observation of elastic and plastic deformations at the Au(111) Surfaces, Phys. Rev. Lett., 52 (1984) 656-658.

[58] L.D. Marks, D.J. Smith, Direct surface imaging in small metal particles, Nature, 303 (1983) 316-317.

[59] L.D. Marks, D.J. Smith, High resolution studies of small particles of gold and silver I. Multiplytwinned particles, Journal of Crystal Growth, 54 (1981) 425-432.

[60] D. Smith, L. Marks, High resolution studies of small particles of gold and silver: II. Single crystals, lamellar twins and polyparticles, Journal of Crystal Growth, 54 (1981) 433-438.

[61] L.D. Marks, A. Howie, Multiply-twinned particles in silver catalysts, Nature, 282 (1979) 196-198.

[62] A. Howie, L.D. Marks, S.J. Pennycook, New Imaging Methods for Catalyst Particles, Ultramicroscopy, 8 (1982) 163-174.

[63] L.D. Marks, Surface structure and energetics of multiply twinned particles, Philosophical Magazine A, 49 (1984) 81-93.

[64] Y. Lin, J. Wen, L. Hu, R.M. Kennedy, P.C. Stair, K.R. Poeppelmeier, L.D. Marks, Synthesis-dependent atomic surface structures of oxide nanoparticles, Phys Rev Lett, 111 (2013) 156101.

[65] Y. Lin, Z. Wu, J. Wen, K.R. Poeppelmeier, L.D. Marks, Imaging the Atomic Surface Structures of $\mathrm{CeO}_{2}$ Nanoparticles, Nano letters, 14 (2014) 191-196.

[66] J.L. Hutchison, N.A. Briscoe, Surface profile imaging of spinel catalyst particles, Ultramicroscopy, 18 (1985) 435-438.

[67] A.K. Datye, D.J. Smith, The Study of Heterogeneous Catalysts by High-Resolution Transmission Electron Microscopy, Catalysis Reviews, 34 (1992) 129-178. 
[68] L.C. Gontard, L.Y. Chang, C.J. Hetherington, A.I. Kirkland, D. Ozkaya, R.E. Dunin-Borkowski, Aberration-corrected imaging of active sites on industrial catalyst nanoparticles, Angew Chem Int Ed Engl, 46 (2007) 3683-3685.

[69] P.L. Hansen, J.B. Wagner, S. Helveg, J.R. Rostrup-Nielsen, B.S. Clausen, H. Topsoe, Atom-resolved imaging of dynamic shape changes in supported copper nanocrystals, Science, 295 (2002) 2053-2055.

[70] D.A. Jefferson, P.J.F. Harris, Direct imaging of an adsorbed layer by high-resolution electron microscopy, Nature, 332 (1988) 617-620.

[71] J.O. Bovin, R. Wallenberg, D.J. Smith, Imaging of atomic clouds outside the surfaces of gold crystals by electron microscopy, Nature, 317 (1985) 47-49.

[72] Y. Lin, J. Wen, L. Hu, R.M. Kennedy, P.C. Stair, K.R. Poeppelmeier, L.D. Marks, Synthesis-dependent atomic surface structures of oxide nanoparticles, Physical Review Letters, 111 (2013) 1-5.

[73] Y. Lin, Z. Wu, J. Wen, K.R. Poeppelmeier, L.D. Marks, Imaging the atomic surface structures of $\mathrm{CeO}_{2}$ nanoparticles, Nano letters, 2 (2014).

[74] C.J. Gilmore, L.D. Marks, D. Grozea, C. Collazo, E. Landree, R.D. Twesten, Direct solutions of the Si(111) 7x7 structure, Surface Science, 381 (1997) 77-91.

[75] L.D. Marks, Rigor, and Plan-View Simulation of Surfaces, Ultramicroscopy, 38 (1991) 325-332.

[76] L.D. Marks, T.S. Savage, J.P. Zhang, R. Ai, Validity of the Kinematical Approximation in Transmission Electron-Diffraction for Surfaces, Revisited, Ultramicroscopy, 38 (1991) 343-347.

[77] L.D. Marks, Registry and UHV transmission electron diffraction of surfaces, Ultramicroscopy, 45 (1992) 145-154.

[78] P. Xu, L.D. Marks, Intensities of Surface Diffraction Spots in Plan View, Ultramicroscopy, 45 (1992) 155-157.

[79] R. Kilaas, MacTempasX, in, 2015.

[80] R. Kilaas, L.D. Marks, C.S. Own, EDM 1.0: electron direct methods, Ultramicroscopy, 102 (2005) 233237.

[81] L.D. Marks, A.N. Chiaramonti, S.U. Rahman, M.R. Castell, Transition from Order to Configurational Disorder for Surface Reconstructions on $\mathrm{SrTiO}_{3}(111)$, Phys Rev Lett, 114 (2015) 226101.

[82] R. Plass, L.D. Marks, UHV Transmission Electron-Microscopy Structure Determination of the Si(111)(V3xV3)-R30-Au Surface, Surface Science, 342 (1995) 233-249.

[83] C. Collazo-Davila, D. Grozea, E. Landree, L.D. Marks, Transmission electron diffraction determination of the Ge(001)- (2x1) surface structure, Surface Science, 375 (1997) 293-301.

[84] C. Collazo-Davila, D. Grozea, L.D. Marks, Determination and refinement of the Ag/Si(111)-(3x1) surface structure, Physical Review Letters, 80 (1998) 1678-1681. 